\title{
Near miss neonatal em hospitais de referência para gestação e parto de alto risco: estudo transversal
}

\author{
Neonatal near miss in referral hospitals for \\ high-risk pregnancy and childbirth: \\ a cross-sectional study
}

\section{Near miss neonatal en hospitales de referencia para el embarazo y parto de alto riesgo: estudio transversal}

Karla Eveline Ximenes de França 1 Mirella Bezerra Rodrigues Vilela 1 Paulo Germano de Frias 2 Milena Ayres Chaves 1 Silvia Wanick Sarinho 1

doi: 10.1590/0102-311X00196220

\section{Resumo}

O objetivo do artigo foi descrever e comparar indicadores de near miss neonatal em hospitais de referência para gestação e parto de alto risco. É um estudo exploratório, transversal, realizado em dois hospitais gerais localizados na cidade do Recife, Pernambuco, Brasil. Considerou-se casos de near miss neonatal os recém-nascidos do ano de 2016 com idade gestacional < 33 semanas ou peso ao nascer $<1.750 \mathrm{~g}$ ou Apgar no 5o minuto de vida $<7$ ou internação em unidade de terapia intensiva (UTI) neonatal, e que permaneceram vivos até 7 dias de vida. Os dados foram extraídos do Sistema de Informações sobre Nascidos Vivos e sobre Mortalidade, do Sistema de Informações Hospitalares e do Cadastro Nacional de Estabelecimentos de Saúde, para caracterizar todos os nascidos vivos das instituições, os casos de near miss e a disponibilidade de tecnologia. Calculou-se os indicadores de near miss neonatal e a taxa de mortalidade neonatal precoce. O Instituto de Medicina Integral Professor Fernando Figueira acolheu a clientela de maior gravidade, apresentou maior taxa de near miss neonatal $(119,21$ por mil nascidos vivos; $p=0,009)$ e de mortalidade neonatal precoce $(35,22$ por mil nascidos vivos; $p<0,001)$. O Hospital das Clínicas registrou a maior proporção de internações em UTI neonatal (76\% dos casos; $p<0,001)$. Os indicadores de near miss neonatal demonstraram diferenças entre os hospitais analisados, sendo úteis para a vigilância da assistência neonatal em instituições de saúde, mas necessitam de atenção ao perfil e contexto local quando a intenção é realizar avaliações classificatórias. Os achados mostram a complexidade de avaliar diferentes serviços de saúde.

Near Miss; Recém-Nascido; Mortalidade Neonatal Precoce; Avaliação em Saúde; Sistemas de Informação
Correspondência

K. E. X. França

Av. Liberdade 440, bloco Juliana Dias, apto. 901, Recife, PE 50920-135, Brasil.

karla_ximenes@hotmail.com

1 Universidade Federal de Pernambuco, Recife, Brasil. 2 Instituto de Medicina Integral Professor Fernando Figueira, Recife, Brasil. 


\section{Introdução}

No período compreendido entre o nascimento e os seis dias de vida, os recém-nascidos são expostos a um maior risco para o óbito infantil e a situações de maior gravidade e/ou quase morte, reconhecidas como near miss neonatal, que constituem um problema de saúde pública no mundo 1,2. A taxa de mortalidade neonatal global decresceu entre 1990 e 2018 de 37/1.000 nascidos vivos para 18/1.000 e no Brasil de 25/1.000 nascidos vivos para 8/1.000 3. Estimativas apontam que existem até 10 vezes mais casos de near miss neonatal do que mortes 4 .

A mortalidade e o near miss neonatal são utilizados para análise e monitoramento da assistência à saúde das gestantes e recém-nascidos por estarem associados às condições de saúde materno-infantil, à qualidade da assistência prestada à mãe durante a gestação, parto, pós-parto e ao neonato no nascimento e durante a internação hospitalar 1,5. Indicadores de mortalidade apresentam definições claras e consensuais, o que não ocorre com o near miss neonatal 6 .

A Organização Mundial da Saúde (OMS) propôs um protocolo com critérios para a identificação de casos de near miss neonatal com base em condições fortemente associadas ao maior risco de morte no período perinatal (prematuridade, baixo peso ao nascer e asfixia), indicadores de disfunção orgânica, clínicos ou laboratoriais e de manejo da gravidade 7 . Atualmente, existe uma multiplicidade de definições que se diferenciam em critérios ou nos pontos de corte. Umas usam critérios pragmáticos, como idade gestacional, peso ao nascer e índice de Apgar no 5o minuto de vida 4,8, e outras se baseiam no manejo dos recém-nascidos, como a necessidade de fototerapia, a realização de cirurgias e/ou em disfunções orgânicas 7,9,10,11. Algumas definições utilizam pontos de corte clássicos em neonatalogia 2,12 e outras não $4,9,11$.

Diante da diversidade de definições, as mais específicas podem apresentar dificuldades operacionais para serem utilizadas no cotidiano dos serviços de saúde ${ }^{4}$. O uso de critérios pragmáticos capazes de captar os neonatos com morbidades neonatais graves que ameaçam à vida possibilita a sua aplicação em contextos socioeconômicos variados e na indisponibilidade de tecnologias relacionadas à assistência 13 .

Estudos sobre o near miss neonatal e seus indicadores oportunizam a avaliação e a vigilância da assistência obstétrica e neonatal em maternidades, além de nortearem decisões de profissionais e gestores para subsidiarem o planejamento adequado de prioridades e recursos para a melhoria da qualidade da atenção às gestantes, parturientes e recém-nascidos 14,15 . Na avaliação de serviços de saúde, o uso de indicadores de mortalidade para comparar hospitais requer cuidados adicionais na análise considerando, entre outros aspectos, o perfil da instituição e da clientela, a gravidade de casos atendidos e a disponibilidade de diferentes tecnologias 16 . O near miss neonatal, por ser uma situação de quase morte e se basear nos mesmos fatores de risco para a mortalidade neonatal, requer cuidados equivalentes. Em contextos em que a morte neonatal é um evento raro (do ponto de vista matemático) o estudo do near miss é útil, pois o número de casos é mais expressivo e com menor repercussão do efeito dos pequenos números, ainda que os cuidados relacionados às particularidades de cada instituição permaneçam necessários 4 .

Alguns estudos sugerem o uso do near miss neonatal em conjunto com a mortalidade institucional para comparar a assistência neonatal em diferentes estabelecimentos de saúde 8,17, desde que de mesma complexidade 12. Porém, são poucos os trabalhos e há incertezas quanto à utilidade do conceito do near miss neonatal e seus indicadores para a avaliação institucional. Este artigo objetivou descrever e comparar indicadores de near miss neonatal em hospitais de referência para gestação e parto de alto risco. 


\section{Métodos}

Estudo exploratório, transversal, desenvolvido em dois hospitais gerais de referência para gestação e parto de alto risco, que prestam serviço exclusivamente ao Sistema Único de Saúde (SUS), localizados na cidade do Recife, Pernambuco, Brasil. O Instituto de Medicina Integral Professor Fernando Figueira (IMIP), uma organização privada sem fins lucrativos, conta com leitos destinados à obstetrícia clínica (28), obstetrícia cirúrgica (76), unidade de terapia intensiva (UTI) obstétrica (12), UTI neonatal (18), Unidade de Cuidado Intermediário Neonatal Convencional (UCINco) (56), UCINco interna (32), que é exclusivamente direcionada aos nascidos vivos na instituição, e Unidade de Cuidado Intermediário Neonatal Canguru (UCINca) (22). O Hospital das Clínicas (HC) da Universidade Federal de Pernambuco tem leitos destinados à obstetrícia clínica (15), obstetrícia cirúrgica (15), UTI neonatal (10) e UCINco (5) (Cadastro Nacional de Estabelecimentos de Saúde. http://cnes.datasus. gov.br/pages/consultas.jsp, acessado em 29/Mai/2020).

Foram classificados como casos de near miss neonatal os recém-nascidos do ano 2016 com idade gestacional $<33$ semanas ou peso ao nascer $<1.750 \mathrm{~g}$ ou índice de Apgar no $5^{\circ}$ minuto de vida $<7$ ou internação em UTI neonatal, que nasceram nas duas instituições e permaneceram vivos até o $7^{\circ}$ dia de vida, conforme definição validada 4 . O período neonatal precoce foi escolhido por representar mais de $75 \%$ de todas as mortes neonatais 18 .

Os dados sobre nascidos vivos foram extraídos do Sistema de Informações sobre Nascidos Vivos (SINASC), exceto as internações em UTI neonatal que foram extraídas do Sistema de Informações Hospitalares do SUS (SIH-SUS) e os sobre óbitos neonatais precoces, do Sistema de Informação sobre Mortalidade (SIM). Essas informações foram fornecidas pela Secretaria de Saúde de Pernambuco.

Inicialmente, foram identificados no SINASC os recém-nascidos que apresentaram as condições de risco estudadas no nascimento. Para o critério internação em UTI neontal, o nome do recém-nascido foi verificado no espelho da Autorização de Internação Hospitalar do SIH-SUS e posteriormente localizado no banco do SINASC. No SIM foram verificados os óbitos neonatais precoces e por meio de um linkage determinístico (identificado pelo número da declaração de nascido vivo que consta na declaração de óbito) foram localizados os óbitos que apresentavam condições de risco ao nascer. Também foi realizada uma busca manual com os não pareados por meio do nome da mãe e o sexo do recém-nascido. Os óbitos pareados entre SIM e SINASC foram retirados do banco de nascidos vivos, o que resultou nos sobreviventes: casos de near miss neonatal. Esse procedimento foi realizado separadamente para cada hospital e posteriormente resultou em um banco único com todos os casos para as análises estatísticas.

Para identificar o perfil da clientela e a gravidade dos casos, foram caracterizados todos os nascidos vivos em cada instituição estudada e os respectivos casos de near miss neonatal por meio de variáveis maternas (idade materna, tipo de gravidez, paridade e número de consultas de pré-natal) e do recém-nascido (sexo, tipo de parto, duração da gestação, peso ao nascer, índice de Apgar no 5o minuto de vida, internação em UTI neonatal e presença de malformações congênitas). A disponibilidade de tecnologia nos hospitais foi descrita por meio do número de leitos (UCINco, UCINca, UTI neonatal, obstetrícia clínica e cirúrgica) e de equipamentos para a manutenção da vida (berços aquecidos, aparelhos de fototerapia, incubadoras, respiradores/ventiladores) que constam no Cadastro Nacional de Estabelecimentos de Saúde. Foi calculada a razão de cada equipamento e leitos por mil nascidos vivos, para o universo de nascimentos vivos de cada instituição, os de baixo peso ao nascer $(<2.500 \mathrm{~g})$ e os prematuros (< 37 semanas). Os casos de near miss também foram caracterizados por critério de entrada para identificar o número de critérios e aqueles que mais contribuíram na classificação dos recém-nascidos como near miss em cada hospital.

Os indicadores de near miss neonatal 8 foram calculados. São eles: taxa de mortalidade neonatal precoce (TMNP): refere-se ao número de óbitos neonatais precoces dividido pelo número total de nascidos vivos multiplicado por 1.000; taxa de near miss neonatal (TNMN): número de casos de near miss neonatal dividido pelo número total de nascidos vivos multiplicado por 1.000; taxa de desfecho neonatal grave (TDNG): número de casos de near miss neonatal mais os óbitos neonatais precoces dividido pelo número total de nascidos vivos multiplicado por 1.000; Índice de sobrevivência neonatal precoce (ISNP) 14: número de recém-nascidos sobreviventes na primeira semana de vida entre aqueles com condições com risco de vida ao nascer dividido pelo número total de recém-nascidos 
com condições de risco de vida ao nascer multiplicado por 100; Índice de mortalidade neonatal precoce (IMNP): número de óbitos de recém-nascidos na primeira semana de vida entre aqueles com condições com risco de vida ao nascer dividido pelo número total de recém-nascidos com condições de risco de vida ao nascer multiplicado por 100.

A coleta de informações, processamento e análises estatísticas ocorreram entre julho de 2018 e abril de 2020. A comparação dos nascidos vivos, dos casos de near miss conforme o local de nascimento e a razão de equipamentos e leitos dos hospitais foi realizada por meio do teste do qui-quadrado de Pearson ou exato de Fisher $(\alpha=5 \%)$, e os indicadores de near miss foram comparados usando-se a razão de prevalência, executados nos programas Microsoft Excel 2019 (https://products.office.com/) e Epi-Info versão 7.2.3.1 (https://www.cdc.gov/epiinfo/index.html).

A pesquisa recebeu aprovação do Comitê de Ética em Pesquisa do Centro de Ciências da Saúde da Universidade Federal de Pernambuco, conforme parecer no 2.773 .429 de 17 de julho de 2018 e Certificado de Apresentação para a Apreciação Ética no 90684418.8.0000.5208.

\section{Resultados}

Em 2016, no IMIP ocorreram 5.679 partos de nascidos vivos, 200 óbitos neonatais precoces (158 com condições de risco ao nascer) e 677 casos de near miss neonatal (3,38 vezes o número de óbitos). No mesmo ano, no HC ocorreram 2.454 partos de nascidos vivos, 25 óbitos neonatais precoces (23 com condições de risco ao nascer) e 243 casos de near miss neonatal (9,72 vezes o número de óbitos).

O perfil epidemiológico dos nascidos vivos nas duas instituições evidenciou diferenças estatisticamente significantes em que o HC apresentou $24,16 \%$ de mães adolescentes, $50 \%$ do total de partos foram cesarianas e $8,03 \%$ dos recém-nascidos necessitaram de internação em UTI neonatal. O IMIP apresentou a maior proporção de gravidez múltipla $(5,62 \%)$ e de mulheres que realizaram de 0 a 3 $(12,92 \%)$ e de 4 a 6 consultas $(36,27 \%)$ de pré-natal, 8,59\% dos recém nascidos nasceram antes de 33 semanas e 20,11\% entre 33 e 36 semanas, 2,76\% tinham peso extremamente baixo ao nascer, 3,63\% peso muito baixo e 18,42\% baixo peso ao nascer, 3,58\% tiveram índice de Apgar no 5 o minuto de vida $<7$ e 4,88\% dos recém-nascidos apresentavam algum tipo de malformação congênita (Tabela 1).

Em relação à disponibilidade de tecnologia nas instituições estudadas, o IMIP apresenta a maior proporção de equipamentos por mil nascidos vivos ( 13 berços aquecidos e 12 incubadoras; $\mathrm{p}<0,001$ ). O HC apresenta o maior número de leitos de UTI neonatal, equipamentos de fototerapia e respiradores na análise da razão por mil recém-nascidos $<2.500 \mathrm{~g}$ e por mil recém-nascidos $<37$ semanas ( $\mathrm{p}$ $<0,001)$ (Tabela 2).

$\mathrm{Na}$ caracterização dos casos de near miss neonatal quanto às variáveis maternas, biológicas e do nascimento, o IMIP apresentou quase o dobro da proporção de mulheres que realizaram de zero a três consultas de pré-natal $(28,51 \%)$ e de prematuros abaixo de 33 semanas de gestação $(57,02 \%)$. Também registrou a maior proporção de presença de malformações congênitas $(8,12 \%)$, peso extremamente baixo ao nascer $(13,14 \%)$ e peso muito baixo ao nascer $(26,14 \%)$ em relação ao HC. Este estabelecimento, em contrapartida, apresentou a maior proporção de casos de near miss com necessidade de internação em UTI neonatal (76,13\%) (Tabela 3).

O IMIP registrou a maior proporção de recém-nascidos classificados como near miss neonatal por três $(23,04 \%)$ ou quatro $(4,73 \%)$ critérios de entrada. O critério que exclusivamente mais classificou casos de near miss foi internação em UTI neonatal e o HC totalizou 47,32\% de todos os casos classificados como near miss por este critério (Tabela 4).

Houve variações nos indicadores de near miss neonatal segundo local de nascimento, com maior taxa de near miss neonatal no IMIP (119,21 por mil nascidos vivos; $\mathrm{p}=0,009)$, que também demonstrou a maior taxa de mortalidade neonatal precoce $(35,22$ por mil nascidos vivos; $\mathrm{p}<0,001)$ e a maior proporção de óbito dentre os recém-nascidos com condições de risco no nascimento (18,92\%; $\mathrm{p}<0,001$ ) (Tabela 5). 
Tabela 1

Perfil epidemiológico dos nascidos vivos no ano de 2016 segundo local de nascimento. Instituto de Medicina Integral Professor Fernando Figueira (IMIP) e Hospital das Clínicas (HC), Recife, Pernambuco, Brasil, 2016.

\begin{tabular}{|c|c|c|c|c|c|c|c|}
\hline \multirow[t]{2}{*}{ Variáveis } & \multicolumn{2}{|c|}{$\begin{array}{c}\text { IMIP } \\
{[N=5.679]}\end{array}$} & \multicolumn{2}{|c|}{$\begin{array}{c}\text { HC } \\
{[\mathrm{N}=2.454]}\end{array}$} & \multicolumn{2}{|c|}{$\begin{array}{c}\text { Total } \\
{[\mathrm{N}=\mathbf{8 . 1 3 3}]}\end{array}$} & \multirow[t]{2}{*}{ Valor de $p$ * } \\
\hline & $\mathbf{n}$ & $\%$ & $n$ & $\%$ & $n$ & $\%$ & \\
\hline \multicolumn{8}{|c|}{ Idade materna (anos) } \\
\hline $10-19$ & 1.207 & 21,25 & 593 & 24,16 & 1.800 & 22,13 & 0,01 \\
\hline $20-35$ & 3.868 & 68,11 & 1.609 & 65,57 & 5.477 & 67,34 & \\
\hline 36 ou mais & 604 & 10,64 & 252 & 10,27 & 856 & 10,53 & \\
\hline \multicolumn{8}{|l|}{ Tipo de gravidez ** } \\
\hline Única & 5.358 & 94,38 & 2.374 & 96,78 & 7.732 & 95,10 & $<0,001$ \\
\hline Dupla ou mais & 309 & 5,62 & 79 & 3,22 & 398 & 4,90 & \\
\hline \multicolumn{8}{|l|}{ Paridade $* * *$} \\
\hline 1 filho & 2.326 & 40,98 & 1.013 & 41,30 & 3.339 & 41,08 & 0,808 \\
\hline 2 filhos ou mais & 3.350 & 59,02 & 1.440 & 58,70 & 4.790 & 58,92 & \\
\hline \multicolumn{8}{|c|}{ Número de consultas de pré-natal } \\
\hline $0-3$ & 734 & 12,92 & 194 & 7,91 & 928 & 11,41 & $<0,001$ \\
\hline $4-6$ & 2.060 & 36,27 & 675 & 27,51 & 2.735 & 33,63 & \\
\hline 7 ou mais & 2.885 & 50,80 & 1.585 & 64,59 & 4.470 & 54,96 & \\
\hline \multicolumn{8}{|l|}{ Sexo \# } \\
\hline Feminino & 2.754 & 48,60 & 1.216 & 49,57 & 3.970 & 48,89 & 0,151 \\
\hline Masculino & 2.913 & 51,40 & 1.237 & 50,43 & 4.150 & 51,11 & \\
\hline \multicolumn{8}{|l|}{ Parto \#\# } \\
\hline Vaginal & 3.102 & 54,63 & 1.227 & 50,00 & 4.329 & 53,23 & $<0,001$ \\
\hline Cesárea & 2.576 & 45,37 & 1.227 & 50,00 & 3.803 & 46,77 & \\
\hline \multicolumn{8}{|c|}{ Duração da gestação (semanas) \#\#\# } \\
\hline$<33$ & 487 & 8,59 & 98 & 4,00 & 585 & 7,21 & $<0,001$ \\
\hline $33-36$ & 1.140 & 20,11 & 354 & 14,47 & 1.494 & 18,41 & \\
\hline$\geq 37$ & 4.043 & 71,31 & 1.995 & 81,53 & 6.038 & 74,39 & \\
\hline \multicolumn{8}{|c|}{ Peso ao nascer (gramas) } \\
\hline$<1.000$ & 157 & 2,76 & 30 & 1,22 & 187 & 2,30 & $<0,001$ \\
\hline 1.000 a 1.499 & 206 & 3,63 & 18 & 0,73 & 224 & 2,75 & \\
\hline 1.500 a 2.499 & 1.046 & 18,42 & 276 & 11,25 & 1.322 & 16,25 & \\
\hline$\geq 2.500$ & 4.270 & 75,19 & 2.130 & 86,80 & 6.400 & 78,69 & \\
\hline \multicolumn{8}{|c|}{ Índice de Apgar no $5 \circ$ minuto $§$} \\
\hline$<7$ & 203 & 3,58 & 36 & 1,47 & 239 & 2,94 & $<0,001$ \\
\hline$\geq 7$ & 5.468 & 96,42 & 2.415 & 98,53 & 7.883 & 97,06 & \\
\hline \multicolumn{8}{|c|}{ Internação em UTI neonatal } \\
\hline Sim & 428 & 7,53 & 197 & 8,03 & 625 & 7,68 & $<0,001$ \\
\hline Não & 5.251 & 92,47 & 2.257 & 91,97 & 7.508 & 92,32 & \\
\hline \multicolumn{8}{|c|}{ Malformações congênitas §§ } \\
\hline $\operatorname{Sim}$ & 277 & 4,88 & 44 & 1,80 & 321 & 3,95 & $<0,001$ \\
\hline Não & 5.399 & 95,12 & 2.407 & 98,20 & 7.806 & 96,05 & \\
\hline
\end{tabular}

UTI: unidade de terapia intensiva.

* Teste qui-quadrado de Pearson; $a=5 \%$;

** 2 casos excluídos IMIP e 1 caso HC: informação ignorada;

*** 3 casos excluídos IMIP e 1 caso HC: informação ignorada;

\# 12 casos excluídos IMIP e 1 caso HC: sexo indeterminado;

\#\# 1 caso excluído IMIP: informação ignorada;

\#\# 9 casos excluídos IMIP e 7 casos HC: informação ignorada;

§ 8 casos excluídos IMIP e 3 casos HC: informação ignorada;

$\S \S 3$ casos excluídos IMIP e HC: informação ignorada. 
Tabela 2

Disponibilidade de tecnologia das instituições por mil nascidos vivos, baixo peso ao nascer e prematuridade. Instituto de Medicina Integral Professor Fernando Figueira (IMIP) e Hospital das Clínicas (HC), Recife, Pernambuco, Brasil, 2016.

\begin{tabular}{|c|c|c|c|}
\hline $\begin{array}{l}\text { Equipamentos para manutenção } \\
\text { da vida e número de leitos }\end{array}$ & IMIP & HC & Valor de $p$ * \\
\hline Berços aquecidos & $N=72$ & $N=14$ & \\
\hline Nascidos vivos ** & 13 & 5 & 0,972 \\
\hline Recém-nascido $<2.500 \mathrm{~g} * \star \star$ & 51 & 43 & $<0,001$ \\
\hline Recém-nascido $<37$ semanas \# & 44 & 31 & $<0,001$ \\
\hline Equipamentos de fototerapia & $N=42$ & $N=16$ & \\
\hline Nascidos vivos ** & 7 & 6 & 0,340 \\
\hline Recém-nascido $<2.500 \mathrm{~g} * \star \star$ & 30 & 49 & $<0,001$ \\
\hline Recém-nascido < 37 semanas \# & 26 & 35 & $<0,001$ \\
\hline Incubadoras & $N=68$ & $N=15$ & \\
\hline Nascidos vivos ** & 12 & 6 & 0,972 \\
\hline Recém-nascido $<2.500 \mathrm{~g} * \star \star$ & 48 & 46 & $<0,001$ \\
\hline Recém-nascido $<37$ semanas \# & 42 & 33 & $<0,001$ \\
\hline Respiradores/ventiladores & $N=18$ & $N=10$ & \\
\hline Nascidos vivos ** & 3 & 4 & $0,256 \# \#$ \\
\hline Recém-nascido $<2.500 \mathrm{~g} * \star \star$ & 13 & 31 & $<0,001$ \\
\hline Recém-nascido $<37$ semanas \# & 11 & 22 & $<0,001$ \\
\hline Leitos de UCINco & $N=88$ & $N=5$ & \\
\hline Nascidos vivos ** & 15 & 2 & $0,150 \# \#$ \\
\hline Recém-nascido $<2.500 \mathrm{~g} * \star \star$ & 62 & 15 & 0,975 \\
\hline Recém-nascido < 37 semanas \# & 54 & 11 & 0,421 \\
\hline Leitos de UCINca & $N=22$ & & \\
\hline Nascidos vivos ** & 4 & - & - \\
\hline Recém-nascido $<2.500 \mathrm{~g} * \star \star$ & 15 & - & - \\
\hline Recém-nascido $<37$ semanas \# & 13 & - & - \\
\hline Leitos de UTI neonatal & $N=18$ & $N=10$ & \\
\hline Nascidos vivos ** & 3 & 4 & $0,256 \# \#$ \\
\hline Recém-nascido $<2.500 \mathrm{~g} * \star \star$ & 13 & 30 & $<0,001$ \\
\hline Recém-nascido $<37$ semanas \# & 11 & 22 & $<0,001$ \\
\hline Leitos de obstetrícia cirúrgica & $N=76$ & $N=15$ & \\
\hline Nascidos vivos ** & 13 & 06 & 0,907 \\
\hline Leitos de obstetrícia clínica & $N=28$ & $N=15$ & \\
\hline Nascidos vivos ** & 5 & 6 & 0,152 \\
\hline
\end{tabular}

UCINca: Unidade de Cuidado Intermediário Neonatal Canguru; UCINco: Unidade de Cuidado Intermediário Neonatal Convencional; UTI: unidade de terapia intensiva.

Fonte: Cadastro Nacional de Estabelecimentos de Saúde; competência dezembro/2016.

* Teste qui-quadrado de Pearson, $a=5 \%$;

** Por mil nascidos vivos (total de nascidos vivos: 5.679 no IMIP e 2.454 no HC);

*** Por mil recém-nascidos < 2.500g (total de recém-nascido < 2.500g: 1.409 no IMIP e 324 no HC);

\# Por mil recém-nascidos < 37 semanas (total de recém-nascido < 37 semanas: 1.627 no IMIP e 452 no HC);

\#\# Teste exato de Fisher. 
Tabela 3

Variáveis maternas, biológicas e do nascimento dos casos de near miss neonatal segundo local de nascimento. Instituto de Medicina Integral Professor Fernando Figueira (IMIP) e Hospital das Clínicas (HC), Recife, Pernambuco, Brasil, 2016.

\begin{tabular}{|c|c|c|c|c|c|c|c|}
\hline \multirow[t]{2}{*}{ Variáveis } & \multicolumn{2}{|c|}{$\begin{array}{c}\text { IMIP } \\
{[\mathrm{N}=677]}\end{array}$} & \multicolumn{2}{|c|}{$\begin{array}{c}\text { HC } \\
{[N=243]}\end{array}$} & \multicolumn{2}{|c|}{$\begin{array}{c}\text { Total } \\
{[\mathrm{N}=920]}\end{array}$} & \multirow[t]{2}{*}{ Valor de $p$ * } \\
\hline & $\mathbf{n}$ & $\%$ & $\mathbf{n}$ & $\%$ & $\mathbf{n}$ & $\%$ & \\
\hline \multicolumn{8}{|c|}{ Idade materna (anos) } \\
\hline 10-19 & 157 & 23,19 & 66 & 27,16 & 223 & 24,24 & 0,43 \\
\hline $20-35$ & 452 & 66,77 & 152 & 62,55 & 604 & 65,65 & \\
\hline 36 ou mais & 68 & 10,04 & 25 & 10,29 & 93 & 10,11 & \\
\hline \multicolumn{8}{|l|}{ Tipo de gravidez } \\
\hline Única & 582 & 85,97 & 215 & 88,48 & 797 & 86,63 & 0,38 \\
\hline Dupla ou mais & 95 & 14,03 & 28 & 11,52 & 123 & 13,37 & \\
\hline \multicolumn{8}{|l|}{ Paridade } \\
\hline 1.filho & 291 & 42,98 & 109 & 44,86 & 400 & 43,48 & 0,54 \\
\hline 2 filhos ou mais & 386 & 57,02 & 134 & 55,14 & 520 & 56,52 & \\
\hline \multicolumn{8}{|c|}{ Número de consultas de pré-natal } \\
\hline $0-3$ & 193 & 28,51 & 37 & 15,22 & 230 & 25,00 & $<0,001$ \\
\hline $4-6$ & 299 & 44,16 & 98 & 40,33 & 397 & 43,15 & \\
\hline 7 ou mais & 185 & 27,33 & 108 & 44,45 & 293 & 31,85 & \\
\hline \multicolumn{8}{|l|}{ Sexo ** } \\
\hline Feminino & 338 & 50,15 & 124 & 51,03 & 462 & 50,38 & 0,87 \\
\hline Masculino & 336 & 49,85 & 119 & 48,97 & 455 & 49,62 & \\
\hline \multicolumn{8}{|l|}{ Parto } \\
\hline Vaginal & 294 & 43,43 & 111 & 45,68 & 405 & 44,02 & 0,59 \\
\hline Cesárea & 383 & 56,57 & 132 & 54,32 & 515 & 55,98 & \\
\hline \multicolumn{8}{|c|}{ Duração da gestação (semanas) *** } \\
\hline$<33$ & 386 & 57,02 & 82 & 33,88 & 468 & 50,92 & $<0,001$ \\
\hline $33-36$ & 170 & 25,11 & 86 & 35,53 & 256 & 27,85 & \\
\hline$\geq 37$ & 121 & 17,87 & 74 & 30,59 & 195 & 21,23 & \\
\hline \multicolumn{8}{|c|}{ Peso ao nascer (gramas) } \\
\hline$<1.000$ & 89 & 13,14 & 18 & 7,41 & 107 & 11,63 & $<0,001$ \\
\hline 1.000 a 1.499 & 177 & 26,14 & 17 & 6,99 & 194 & 21,08 & \\
\hline 1.500 a 2.499 & 269 & 39,73 & 90 & 37,03 & 359 & 39,02 & \\
\hline$\geq 2.500$ & 142 & 20,99 & 118 & 48,57 & 260 & 28,27 & \\
\hline \multicolumn{8}{|c|}{ Índice de Apgar no 5 o minuto \# } \\
\hline$<7$ & 106 & 15,73 & 26 & 10,70 & 132 & 14,39 & 0,07 \\
\hline$\geq 7$ & 568 & 84,27 & 217 & 89,30 & 785 & 85,61 & \\
\hline \multicolumn{8}{|c|}{ Internação em UTI neonatal } \\
\hline Sim & 355 & 52,44 & 185 & 76,13 & 540 & 58,70 & $<0,001$ \\
\hline Não & 322 & 47,56 & 58 & 23,87 & 380 & 41,30 & \\
\hline \multicolumn{8}{|c|}{ Malformações congênitas } \\
\hline Sim & 55 & 8,12 & 8 & 3,29 & 63 & 6,85 & 0,016 \\
\hline Não & 622 & 91,88 & 235 & 96,71 & 857 & 93,15 & \\
\hline
\end{tabular}

UTI: unidade de terapia intensiva neonatal.

* Teste qui-quadrado de Pearson; $a=5 \%$;

** 3 casos excluídos IMIP: sexo indeterminado;

*** 1 caso excluído HC: informação ignorada;

\# 3 casos excluídos IMIP: informação ignorada. 
Tabela 4

Caracterização dos casos de near miss neonatal pelo número de critérios de entrada segundo local de nascimento. Instituto de Medicina Integral Professor Fernando Figueira (IMIP) e Hospital das Clínicas (HC), Recife, Pernambuco, Brasil, 2016

\begin{tabular}{|c|c|c|c|c|c|}
\hline \multirow[t]{2}{*}{ Número de critérios } & \multicolumn{2}{|c|}{$\begin{array}{c}\text { IMIP } \\
{[N=677]}\end{array}$} & \multicolumn{2}{|c|}{$\begin{array}{c}\mathrm{HC} \\
{[\mathrm{N}=243]}\end{array}$} & \multirow[t]{2}{*}{ Valor de $p$ * } \\
\hline & $\mathbf{n}$ & $\%$ & $\mathbf{n}$ & $\%$ & \\
\hline 1 critério ** & 334 & 49,33 & 166 & 68,3 & $<0,001$ \\
\hline Internação em UTI neonatal & 105 & 15,51 & 115 & 47,32 & $<0,001$ \\
\hline Idade gestacional $<33$ semanas & 102 & 15,06 & 31 & 12,76 & 0,44 \\
\hline Peso ao nascer $<1.750 \mathrm{~g}$ & 91 & 13,29 & 8 & 3,29 & $<0,001$ \\
\hline Apgar no 5 o minuto $<7$ & 36 & 5,17 & 12 & 4,93 & 0,97 \\
\hline 2 critérios & 155 & 22,90 & 43 & 17,7 & 0,109 \\
\hline 3 critérios & 156 & 23,04 & 32 & 13,18 & 0,001 \\
\hline 4 critérios & 32 & 4,73 & 2 & 0,82 & 0,01 \\
\hline
\end{tabular}

UTI: unidade de terapia intensiva.

* Teste qui-quadrado de Pearson, $a=5 \%$;

** Casos classificados como near miss neonatal exclusivamente por 1 critério.

\section{Tabela 5}

Comparativo dos indicadores de near miss neonatal segundo local de nascimento. Instituto de Medicina Integral Professor Fernando Figueira (IMIP) e Hospital das Clínicas (HC), Recife, Pernambuco, Brasil, 2016.

\begin{tabular}{lccc}
\hline Indicadores & IMIP & HC & Valor de $\mathbf{p}$ * \\
\hline Taxa de mortalidade neonatal precoce ** & 35,22 & 10,19 & $<0,001$ \\
Taxa de near miss neonatal ** & 119,21 & 99,02 & 0,009 \\
Taxa de desfecho neonatal grave ** & 154,43 & 109,21 & $<0,001$ \\
Índice de sobrevivência neonatal precoce (\%) & 81,08 & 91,35 & $<0,001$ \\
Índice de mortalidade neonatal precoce (\%) & 18,92 & 8,65 & $<0,001$ \\
\hline
\end{tabular}

Nota: total de nascidos vivos em 2016: 5.679 no IMIP; 2.454 no HC.

* Razão de prevalência.

** Por mil nascidos vivos.

\section{Discussão}

Os indicadores de near miss neonatal variaram entre os hospitais analisados e, em geral, aquele que atende o público com perfil de elevado risco para a morte de recém-nascido, acolhe uma clientela que apresenta a maior gravidade e dispõe de maior densidade tecnológica apresentou indicadores mais desfavoráveis. Os indicadores de near miss são úteis para o monitoramento e a vigilância da assistência nas instituições, além de avaliações formativas, entretanto, requerem atenção particular ao se pretender realizar avaliações classificatórias acerca da assistência neonatal para evitar conclusões inadvertidas. Os achados explicitam a complexidade de avaliar diferentes instituições de saúde.

A análise do perfil epidemiológico dos atendidos nos hospitais expressa o perfil das instituições. O hospital de maior porte (IMIP) é referência para casos de malformações congênitas e cirurgia nesta área, com mais que o dobro de nascidos vivos que o outro, e atende à clientela de maior gravidade. As 
variáveis que foram estatisticamente significantes neste estudo são fatores de risco para a morbidade e mortalidade neonatal bastante estudados 5,19. Uma metanálise de estudos observacionais evidenciou maior risco de óbito neonatal quanto à gravidez múltipla, idade gestacional $<37$ semanas, peso ao

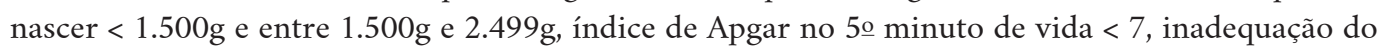
pré-natal ou sua ausência e malformações congênitas 20. Essa última é um fator de risco importante e a segunda maior causa de mortalidade neonatal no Brasil 5,19. Um estudo em 15 municípios de uma região de saúde do Estado de Minas Gerais mostrou que 42,8\% dos óbitos infantis tiveram como causa básica as malformações congênitas 20 , e uma coorte no México demonstrou mortalidade neonatal de $32 \%$ destes casos 21 .

Os resultados deste trabalho mostraram variação na razão de equipamentos e leitos por prematuros e baixo peso ao nascer entre as instituições, apesar de a maior disponibilidade absoluta estar no IMIP, indicando a influência do perfil institucional sobre o indicador. Um estudo que analisou a estrutura de 266 maternidades observou que as regiões Norte/Nordeste apresentam a maior deficiência na disponibilidade de equipamentos essenciais e estratégicos para viabilizar a sobrevivência do recém-nascido nas emergências (apenas $45 \%$ dos hospitais públicos tinham todos os equipamentos) 22. No ano analisado neste trabalho, Recife apresentava 2,2 leitos de UCI (UCINco e UCINca) e 1,0 de UTI neonatal para mil nascidos vivos na região, e o parâmetro é de 3,0 e 2,0 por mil nascidos vivos, respectivamente 23 . Parâmetros de base populacional para a análise de instituições requerem atenção particular, em especial as localizadas em polos médicos e que são referência para a região de saúde. Nesse caso, a análise deve se basear na população de cobertura e não exclusivamente no município onde estão sediadas.

A evitabilidade das mortes infantis é muito estudada e há classificações direcionadas à sua análise. Entretanto, a maioria e as mais utilizadas no Brasil não são específicas para o período neonatal, ainda que o inclua. Essas em geral enfatizam o momento do ciclo gravídico puerperal em que o problema básico de saúde ocorreu e quando as intervenções do sistema de saúde poderiam contribuir mais efetivamente para evitar as mortes 24 . Estudos sinalizam que $71 \%$ dos óbitos neonatais são evitáveis a partir do acesso e da garantia de assistência de qualidade no pré-natal, parto e puerpério e da adesão às práticas de cuidado baseadas em evidências 25,26 . Uma pesquisa em serviços públicos de saúde no Sudeste do Brasil observou aumento das taxas de mortalidade devido a causas básicas de óbitos por afecções maternas que afetam o feto e o recém-nascido, que ocorreu provavelmente pela inadequada atenção pré-natal 27 . Em Recife, 70\% dos óbitos poderiam ter sido prevenidos se houvesse adequada atenção à mulher na gestação, o que reforça a importância do pré-natal 28.

Em relação às práticas de cuidado com o recém-nascido, o contato pele a pele do Método Mãe Canguru estimula o aleitamento materno em livre demanda, promove estabilidade térmica e reduz a necessidade de utilização de equipamentos como incubadoras, contribuindo para a alta hospitalar, o crescimento e o desenvolvimento de bebês prematuros e com baixo peso ao nascer 29 . Garantir intervenções e rotinas relacionadas à continuidade do cuidado que atendam as necessidades de gestantes e recém-nascidos é essencial para melhorar a qualidade da assistência materna e neonatal, principalmente em países em desenvolvimento 25 .

A análise dos casos de near miss neonatal mostrou o reflexo do perfil epidemiológico dos nascimentos nas instituições, assinalando uma maior gravidade dos casos provenientes do IMIP. Um trabalho realizado em seis maternidades públicas no Sudeste do Brasil verificou associação do hospital de nascimento e da falta de assistência pré-natal com o near miss neonatal. Essa última também esteve associada a um aumento significativo do risco de morte neonatal 30 . Numa pesquisa que analisou desigualdades regionais no acesso e qualidade da atenção ao pré-natal e ao parto nos serviços públicos de saúde no Brasil, a Região Nordeste apresentou desfechos neonatais desfavoráveis principalmente em relação à prematuridade espontânea, baixo peso ao nascer e restrição do crescimento intrauterino. A peregrinação para o parto esteve associada com o near miss neonatal com razão de chance de 1,48 (IC95\%: 1,23-1,78) 31. O pré-natal realizado de forma adequada contribui para a redução de fatores evitáveis, como a prematuridade e o baixo peso ao nascer 19. Portanto, a melhoria da sua qualidade tem potencial para reduzir a taxa de morbimortalidade neonatal no Brasil 31.

A presença de malformações congênitas é usada como critério de classificação de casos de near miss neonatal em algumas definições 10,12. Uma delas, oriunda de estudo na base de dados da Pesquisa Nascer no Brasil, utilizou os critérios pragmáticos (peso ao nascer, Apgar e idade gestacional), necessi- 
dade de ventilação mecânica e presença de malformações congênitas (de qualquer tipo) 12. Entretanto, outro trabalho alerta para a necessidade de padronizar a definição e para a relevância de classificar os grupos de gravidade da malformação congênita de acordo com a Classificação Internacional de Doenças ao utilizar essa variável como um critério de identificação do near miss neonatal 6 . Uma validação concorrente entre três definições de near miss neonatal, dentre elas a que usa a presença de malformações congênitas 12, mostrou que a definição utilizada neste estudo apresentou resultados equivalentes às demais 4 .

O HC apresentou a maior proporção de internações em UTI neonatal e este critério foi o que exclusivamente mais classificou os recém-nascido como casos de near miss neonatal. O contexto institucional parece estar relacionado a esse achado. Nesse ano em questão, a UCINCo foi desativada temporariamente, a instituição passou a admitir exclusivamente gestantes de baixo risco e os recémnascidos que precisassem de intervenção iam para a UTI neonatal. Esse problema organizacional refletiu em uma maior classificação de casos de near miss por esse critério. Essa situação demostra que o near miss pode ser uma ferramenta de vigilância da assistência ao recém-nascido, que gera um alerta para a necessidade de analisar o contexto dos estabelecimentos de saúde com maior profundidade 14.

A análise dos indicadores de near miss neonatal permitiu observar que o IMIP apresentou mais casos de near miss e os piores desfechos que o $\mathrm{HC}$, incluindo a maior taxa de mortalidade neonatal precoce, o que sinaliza que restringir a análise ao número de sobreviventes pode gerar interpretações equivocadas. O IMNP verifica os óbitos neonatais precoces em relação aos recém-nascidos com condições de risco ao nascer. Conforme a pesquisa que sugeriu comparar as diferentes instituições por meio de indicadores de near miss, um baixo índice indicaria alta qualidade da assistência neonatal e um alto índice indicaria que algo poderia ser melhorado 8 . Neste estudo, a análise isolada dos indicadores não aponta para a maior proporção de internações em UTI neonatal do HC, o que interferiu na maior classificação de casos de near miss neonatal. Aspectos organizacionais relacionados ao contexto local do período podem alterar os achados de indicadores de near miss 14 .

Esta pesquisa exploratória apresenta reflexões e levanta hipóteses. Apesar do near miss neonatal ser bastante estudado, ainda não há consenso sobre uma definição padrão, os critérios de classificação, os pontos de corte, o tempo de acompanhamento dos casos (até 7 ou 28 dias) e sobre o seu potencial de utilização como ferramenta de avaliação da qualidade em saúde 6,14,17. A escolha da definição dos casos de near miss neonatal pode modificar os desfechos a depender dos critérios, dos pontos de corte utilizados e do perfil das instituições. Ainda que estudos comparativos entre múltiplas definições mostrem que não há diferenças estatisticamente significantes entre as definições testadas, o uso de critérios pragmáticos e a obtenção de dados dos sistemas de informações em saúde oficiais facilitam a operacionalização conceitual do near miss neonatal para fins clínicos e epidemiológicos principalmente diante de recursos tecnológicos menos adequados 4,13. Entretanto, há situações em que é mais pertinente usar uma definição mais específica, sobretudo quando os serviços de saúde não dispõem de capacidade operacional para monitorar um número grande de casos 4.

Para contornar esse problema foi utilizada uma definição validada 4 aplicada em serviços de saúde da mesma complexidade, conforme recomendação da literatura 12 . Esta pesquisa usou dados secundários de sistemas oficiais que podem acarretar em viés de informação. Porém, o Estado de Pernambuco tem dados de nascidos vivos e mortalidade com boa qualidade, cobertura e completude 32,33.

O near miss neonatal pode ser uma ferramenta útil para monitorar a assistência neonatal em diferentes instituições, desde que sejam comparados estabelecimentos de saúde de complexidade similar e haja uma análise do perfil das instituições, da clientela, da gravidade dos casos e da disponibilidade de diferentes tecnologias para identificar situações de alerta que precisem ser modificadas, tornando-se, portanto, um instrumento de vigilância em saúde que pode nortear gestores nas tomadas de decisão. 


\section{Colaboradores}

K. E. X. França contribuiu na concepção, projeto, análise e interpretação dos dados, redação do artigo, aprovação final da versão a ser publicada. M. B. R. Vilela contribuiu na análise e interpretação dos dados, revisão crítica relevante do conteúdo intelectual, aprovação final da versão a ser publicada. P. G. Frias e S. W. Sarinho contribuíram na concepção e projeto, revisão crítica relevante do conteúdo intelectual, aprovação final da versão a ser publicada. $M$. A. Chaves contribuiu na análise e interpretação dos dados, redação do artigo, aprovação final da versão a ser publicada.

\section{Informações adicionais}

ORCID: Karla Eveline Ximenes de França (00000002-4090-953X); Mirella Bezerra Rodrigues Vilela (0000-0001-5113-7144); Paulo Germano de Frias (0000-0003-4497-8898); Milena Ayres Chaves (0000-0002-6572-9404); Silvia Wanick Sarinho (0000-0002-2556-3323).

\section{Referências}

1. Garcia LP, Fernandes CM, Traebert J. Risk factors for neonatal death in the capital city with the lowest infant mortality rate in Brazil. J Pediatr (Rio J.) 2019; 95:194-200.

2. Shroff BD, Ninama NH. A call for eminence obstetrics care by way of "neonatal near miss" events (NNM): a hospital-based case-control study. J Obstet Gynecol India 2019; 69:50-5.

3. World Health Organization. Levels \& trends in child mortality: report 2019. Estimates developed by the UN Inter-agency Group for Child Mortality Estimation. Geneva: World Health Organization; 2019.

4. França KEX, Vilela MBR, Frias PG, Gaspar GS, Sarinho SW. Near miss neonatal precoce identificado com base em sistemas de informação em saúde. Cad Saúde Pública 2018; 34:e00167717.

5. Lansky S, Friche AAL, Silva AAM, Campos D, Bittencourt SDA, Carvalho ML, et al. Pesquisa Nascer no Brasil: perfil da mortalidade neonatal e avaliação da assistência à gestante e ao recém-nascido. Cad Saúde Pública 2014; 30 Suppl:S192-207.

6. Santos J, Cecatti J, Serruya S, Almeida P, Duran P, Mucio B, et al. Neonatal Near Miss: the need for a standard definition and appropriate criteria and the rationale for a prospective surveillance system. Clinics 2015; 70:820-6.

7. Souza JP, Gülmezoglu AM, Carroli G, Lumbiganon P, Qureshi Z. The world health organization multicountry survey on maternal and newborn health: study protocol. BMC Health Serv Res 2011; 11:286.

8. Pileggi C, Souza JP, Cecatti JG, Faúndes A. Neonatal near miss approach in the 2005 WHO Global Survey Brazil. J Pediatr (Rio J.) 2010; 86:21-6.

9. Bakari A, Bell AJ, Oppong SA, Bockarie Y, Wobil P, Plange-Rhule G, et al. Neonatal nearmisses in Ghana: a prospective, observational, multi-center study. BMC Pediatr 2019; 19:509.

10. Mersha A, Bante A, Shibiru S. Factors associated with neonatal near-miss in selected hospitals of Gamo and Gofa zones, southern Ethiopia: nested case-control study. BMC Pregnancy Childbirth 2019; 19:516.

11. Pileggi-Castro C, Camelo JS, Perdoná GC, Mussi-Pinhata MM, Cecatti JG, Mori R, et al. Development of criteria for identifying neonatal near-miss cases: analysis of two WHO multicountry cross-sectional studies. BJOG 2014; 121 Suppl 1:110-8.

12. Silva AAM, Leite ÁJM, Lamy ZC, Moreira MEL, Gurgel RQ, Cunha AJLA, et al. Morbidade neonatal near miss na pesquisa Nascer no Brasil. Cad Saúde Pública 2014; 30 Suppl: S182-91.

13. Kale PL, Jorge MHPM, Laurenti R, Fonseca SC, Silva KS. Pragmatic criteria of the definition of neonatal near miss: a comparative study. Rev Saúde Pública 2017; 51:111. 
14. França KEX, Vilela MBR, Frias PG, Sarinho SW. Early neonatal near miss in a University Hospital: comparative cross-sectional study. Rev Paul Pediatr 2021; 39:e2019317.

15. Silva GA, Rosa KA, Saguier ESF, Henning E, Mucha F, Franco SC. A populational based study on the prevalence of neonatal near miss in a city located in the South of Brazil: prevalence and associated factors. Rev Bras Saúde Matern Infant 2017; 17:169-77.

16. Travassos C, Noronha JC, Martins M. Mortalidade hospitalar como indicador de qualidade: uma revisão. Ciênc Saúde Colet 1999; 4:36781.

17. Brasil DRPA, Vilela MBR, França KEX, Sarinho SW. Neonatal morbidity near miss in tertiary hospitals in a capital of Northeast Brazil. Rev Paul Pediatr 2019; 37:275-82.

18. Carlo WA, Travers CP. Maternal and neonatal mortality: time to act. J Pediatr (Rio J.) 2016; 92:543-5.

19. Veloso FCS, Kassar LML, Oliveira MJC, Lima THB, Bueno NB, Gurgel RQ, et al. Analysis of neonatal mortality risk factors in Brazil: a systematic review and meta-analysis of observational studies. J Pediatr (Rio J) 2019; 95:51930.

20. Silva PLN, Costa AA, Farias HMT, Rocha LMF, Oliveira MA, Damasceno RF. Evitabilidade da mortalidade infantil na região de saúde de Janaúba/Monte Azul, Minas Gerais, Brasil. Rev Ciênc Méd Biol 2018; 6:35-41.

21. Reyesa JCL, Ramírez ROP, Ramosa LL, Ruiz LMG, Vázquez EAB, Patinõ VR. Neonatal mortality and associated factors in newborn infants admitted to a Neonatal Care Unit. Arch Argent Pediatr 2018; 116:42-8.

22. Bittencourt SDA, Reis LGC, Ramos MM, Rattner D, Rodrigues PL, Neves DCO, et al. Estrutura das maternidades: aspectos relevantes para a qualidade da atenção ao parto e nascimento. Cad Saúde Pública 2014; 30 Suppl:S208-19.

23. Lima SS, Braga MC, Vanderlei LCM, Luna CF, Frias PG. Avaliação do impacto de programas de assistência pré-natal, parto e ao recém-nascido nas mortes neonatais evitáveis em Pernambuco, Brasil: estudo de adequação. Cad Saúde Pública 2020; 36:e00039719.

24. Dias BAS, Santos Neto ET, Andrade MAC. Classification systems for avoidability of infant deaths: different methods, different repercussions? Cad Saúde Pública 2017; 33:e00125916.
25. Ehret DY, Patterson JK, Bose CL. Improving neonatal care: a global perspective. Clin Perinatol 2017; 44:567-82.

26. Gaiva MAM, Fujimori E, Sato APS. Mortalidade neonatal: análise das causas evitáveis. Rev Enferm UERJ 2015; 23:247-53.

27. Saltarelli RMF, Prado RR, Monteiro RA, Malta DC. Tendência da mortalidade por causas evitáveis na infância: contribuições para a avaliação de desempenho dos serviços públicos de saúde da Região Sudeste do Brasil. Rev Bras Epidemiol 2019; 22:e190020.

28. Marques LJP, Pimentel DR, Oliveira CM, Vilela MBR, Frias PG, Bonfim CV. Concordância da causa básica e da evitabilidade dos óbitos infantis antes e após a investigação no Recife, Pernambuco, 2014. Epidemiol Serv Saúde 2018; 27:e20170557.

29. Zirpoli DB, Mendes RB, Reis TS, Barreiro MSC, Menezes AF. Benefits of the Kangaroo Method: an integrative literature review. Rev Pesqui Cuid Fundam (Online) 2019; 11(2, n. esp): 547-54.

30. Kale PL, Mello-Jorge MHP, Silva KS, Fonseca SC. Neonatal near miss and mortality: factors associated with life-threatening conditions in newborns at six public maternity hospitals in Southeast Brazil. Cad Saúde Pública 2017; 33:e00179115.

31. Leal MC, Esteves-Pereira AP, Viellas EF, Domingues RMSM, Gama SGN. Prenatal care in the Brazilian public health services. Rev Saúde Pública 2020; 54:8.

32. Szwarcwald CL, Leal MC, Esteves-Pereira AP, Almeida WS, Frias PG, Damacena GN, et al. Avaliação das informações do Sistema de Informações sobre Nascidos Vivos (SINASC), Brasil. Cad Saúde Pública 2019; 35:e00214918.

33. Figueirôa $B Q$, Frias $P G$, Vanderlei LCM, Vidal SA, Carvalho PI, Pereira CCB, et al. Evaluation of the implantation of the Mortality Information System in Pernambuco state, Brazil, in 2012. Epidemiol Serv Saúde 2019; 28:2018384. 
Abstract

The article aimed to describe and compare indicators of neonatal near miss in referral hospitals for high-risk pregnancy and childbirth. This exploratory study was performed in two general hospitals in Recife, Pernambuco, Brazil. The study included cases of neonatal near miss in the year 2016 with gestational age < 33 weeks, birthweight $<1,750$, 5-minute Apgar < 7, or admission to the neonatal intensive care unit (ICU) and that remained alive at 7 days of life. Data were extracted from the Brazilian Information System on Live Births, Mortality Information System, Hospital Information System, and National Registry of Healthcare Establishments to characterize all live births at the institutions, cases of near miss, and availability of technology. Neonatal near miss and early neonatal mortality rates were calculated. The Pernambuco Maternal-Child Institute (IMIP) received the most serious cases and had the highest neonatal near miss rate (119.21 per thousand live births; $p=0.009$ ) and early neonatal mortality rate (35.22 per thousand live births; $p<0.001$ ). The University Hospital had the highest proportion of neonatal ICU admissions (76\% of the near miss cases; $p<0.001)$. Neonatal near miss rates differed between the hospitals and are useful for surveillance of neonatal care in healthcare institutions, but they require attention to the local profile and context when the objective is to perform evaluations with classification. The findings reflect the complexity of assessing different health services.

Healthcare Near Miss; Newborn Infant; Early Neonatal Mortality; Health Evaluation; Information Systems

\section{Resumen}

El objetivo del artículo fue describir y comparar indicadores de near miss neonatal en hospitales de referencia para el embarazo y parto de alto riesgo. Se trata de un estudio exploratorio, transversal, realizado en dos hospitales generales, localizados en la ciudad de Recife, Pernambuco, Brasil. Se consideraron casos de near miss neonatal a los recién nacidos del año 2016, con una edad gestacional < 33 semanas o peso al nacer $<1.750 \mathrm{~g}$ o Apgar en el 5 o minuto de vida $<7$ o internamiento en la unidad de terapia intensiva (UTI) neonatal y que permanecieron vivos hasta los 7 días de vida. Los datos se extrajeron del Sistema de Información sobre Nacidos Vivos y sobre Mortalidad, del Sistema de Información Hospitalaria y Registro Nacional de Establecimientos de Salud para caracterizar a todos los nacidos vivos de las instituciones, los casos de near miss y la disponibilidad de tecnología. Se calcularon los indicadores de near miss neonatal y la tasa de mortalidad neonatal precoz. El Instituto de Medicina Integral Profesor Fernando Figueira acogió a pacientes de mayor gravedad, presentó una mayor tasa de near miss neonatal (119,21 por mil nacidos vivos; $p=0,009) y$ de mortalidad neonatal precoz $(35,22$ por mil nacidos vivos; $p<0,001)$. El Hospital de las Clínicas obtuvo mayor proporción de internamientos en UTI neonatal (76\% de los casos de near miss; $p<0,001$ ). Los indicadores de near miss neonatal demostraron diferencias entre los hospitales analizados, siendo útiles para la vigilancia de la asistencia neonatal en instituciones de salud, pero que necesitan atención respecto al perfil y contexto local, cuando la intención es realizar evaluaciones clasificatorias. Los resultados muestran la complejidad de evaluar diferentes servicios de salud.

Near Miss Salud; Recién Nacido; Mortalidad

Neonatal Precoz; Evaluación en Salud;

Sistemas de Información
Recebido em 07/Jul/2020

Versão final reapresentada em 26/Out/2020

Aprovado em 06/Nov/2020 